\title{
Minimally Invasive Approach to Visceral Microsurgical
}

\section{Autoflaps}

\author{
Igor Reshetov $^{1,2}$, Mikhail Ratushnyy ${ }^{1}$ and Victoria Shcherbina ${ }^{2}$ \\ 1. Department of Microsurgery, P.A. Hertzen Moscow Oncological Research Institute, Moscow 125284, Russia \\ 2. Institute for Advanced Studies of FMBA of Russia, Moscow 125424, Russia
}

\begin{abstract}
Organ-preserving and function-sparing treatment, which provides reduction of the duration of treatment and rehabilitation periods, is a promising direction in clinical oncology. Such approach requires application of the most modern, effective and high-tech method of reconstructive and plastic surgery, which is the microsurgical autotransplantation of tissues and organs. The method of minimally invasive abdominal approach to visceral autoflaps in cancer patients was developed. Thirty one male and twenty two female patients with malignant tumors of craniofacial (27) and oropharingeal (26) localization underwent reconstructive surgery after tumor resection. Visceral autoflaps were recovered using minilaparotomy. The minilaparotomy was successful in 50 cases. Twenty six omental flaps, seven gastro-omental flaps, fifteen colonic-omental flaps and five small intestinal flaps were formed and prepared for transplantation. No intra- or postoperative complications associated with abdominal organs were observed. The use of minimally invasive techniques to form visceral autotransplants for reconstruction of head and neck organs, provides significant reduction of surgical trauma and shortening of treatment duration. This method should be indicated for impaired cancer patients or young patients willing to avoid an additional scar in the donor area.
\end{abstract}

Key words: Head and neck cancer, head and neck reconstruction, visceral flaps, microsurgery, autoflaps, video assistance, minimally invasive abdominal approach.

\section{Introduction}

Surgery for tumors of digestive tract, head, neck, etc., may be very traumatic and disabling for a patient, resulting in isolation from family, social networks and participation in everyday life [1, 2]. The possibility of having oro-, pharingo-, esophago- and tracheostomas, salivation, tube feeding, etc., causes irreparable psychological damage to patient's personality, and in some cases, forces to refuse the required radical treatment [3].

Currently, one of the promising directions of oncology is performing reconstructive interventions on the stages of combined and/or complex treatment of patients with malignant tumors. Regarding this, the time of treatment is considerably reduced and the

Corresponding author: Igor Reshetov, M.D., professor, research fields: oncology, head and neck tumors, reconstructive surgery, microsurgery and tissue engineering. E-mail: reshetoviv@mail.ru. quality of patient's rehabilitation is improved, and additionally the degree of disability in the patients who underwent surgery, is also decreased.

Obviously, the most promising direction in clinical oncology is organ-preserving and function-sparing treatment, which provides reduction of the treatment duration and rehabilitation periods. Such kind of approach requires application of the most modern, effective and high-tech method of reconstructive and plastic surgery, which is the microsurgical autotransplantation of tissues and organs [4].

Another promising direction in modern oncology is minimally invasive diagnostics and surgery of tumors of different localization and morphological structure. The method of minimally invasive abdominal approach for formation of visceral autoflaps in cancer patients was developed in the P.A. Hertzen Moscow Cancer Research Institute. 


\section{Materials and Methods}

The presented report is based on the clinical experience of treatment of 53 cancer patients in the age from 16 to 63 years (Table 1). Thirty one male and twenty two female patients (Table 1 ) had malignant tumors of craniofacial (27) and oropharingeal (26) localization. Seventeen patients had tumors of the scalp, 5-tumors of the maxillary area, 5-tumors of the ethmoidal labyrinth, 4 - cancer of the oral cavity floor, 5-tongue cancer, 3-oropharingeal cancer, 8-laryngopharingeal cancer, 1-soft tissue sarcoma of the face, 1-sarcoma of mandible, and 4-parotid salivary gland cancer.

The chosen periomphalic $2 / 3$ circle incision, provided an adequate mono-approach to the abdominal cavity, and was associated with minimal external trauma of the anterior abdominal wall (Fig. 1).

Then the dissection of the aponeurosis in the midline, laparotomy and revision of the abdominal cavity was performed with the video-assistant support (video

Table 1 Distribution of patients by age and sex.

\begin{tabular}{lll}
\hline \multirow{2}{*}{ Age } & \multicolumn{2}{c}{ Sex } \\
\cline { 2 - 3 } & Male & Female \\
\hline$<40$ & 18 & 11 \\
$40-60$ & 13 & 9 \\
$>60$ & 0 & 2 \\
Total & 31 & 22 \\
\hline
\end{tabular}

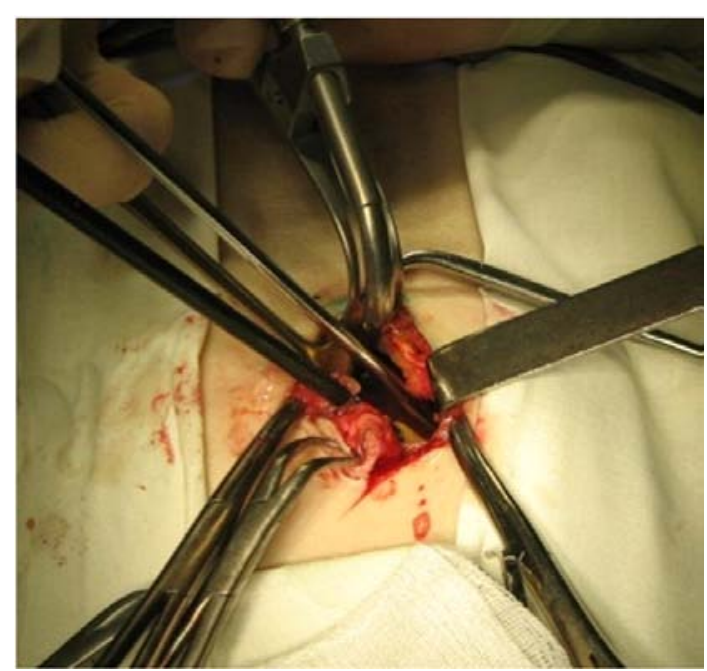

(a)

Fig. 2 Laparotomy and revision of the abdominal cavity. (a) Minilaparotomy; (b) Abdominal revision with the assistance of endoscopic equipment.

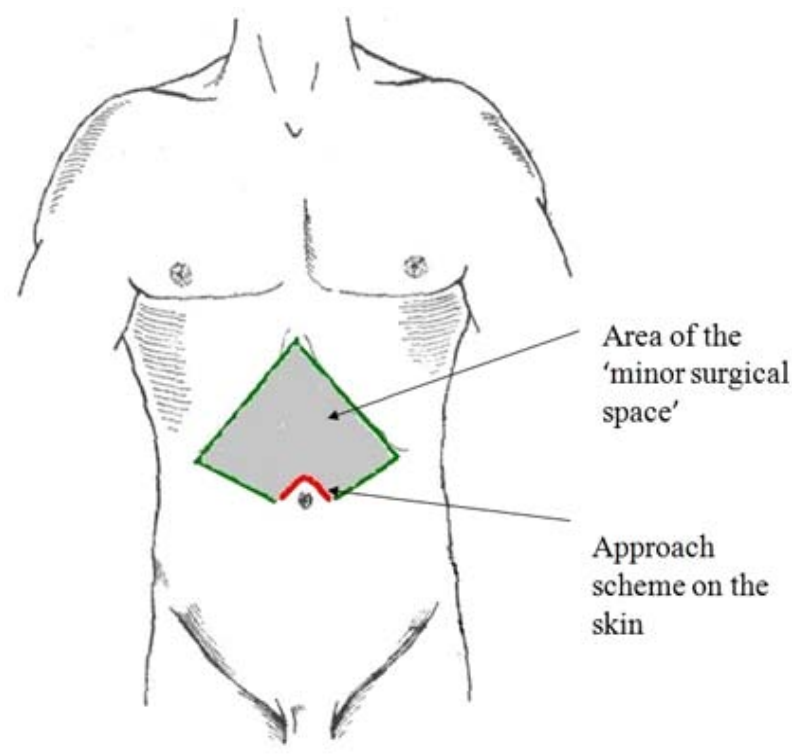

Fig. 1 Marking of the periomphalic approach.

endoscopic rank and tools) (Fig. 2). It was essential to abandon gas-laparoscopy in order to create the "minor surgical space”, using retractors with illumination. This permits to carry out the abdominal revision and accurate mobilization of the potential autoflap through the minimal mono-approach.

Further, the dissection of the adhesions in the abdominal cavity was performed, if necessary. Donor organs (omentum, greater curvature of the stomach, transverse colon, and small intestine) were recovered though the minilaparotomy opening on the anterior abdominal wall. The mobilization of the vascular pedicle

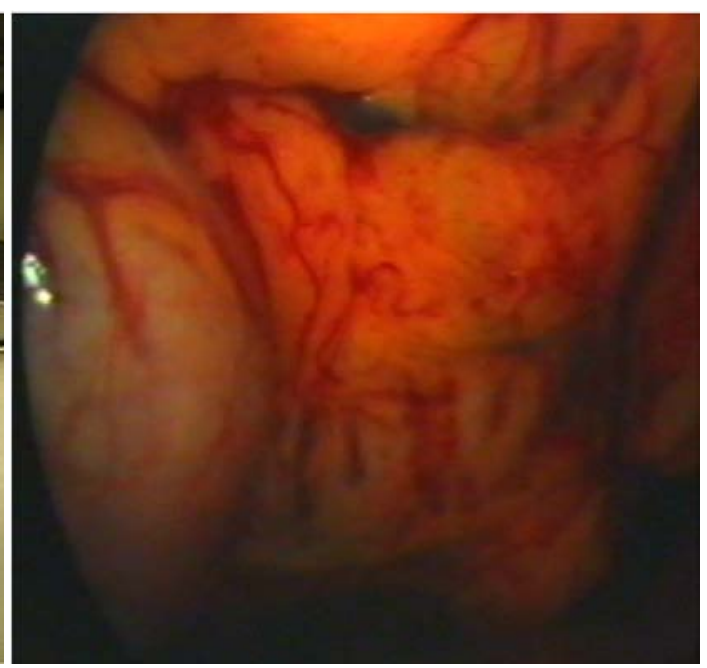

(b) 
(right gastroepiploic vessels, medial colonic vessels, and jejunal vessels) and forming of the visceral graft were performed afterwards. After forming and dissection of the graft, organ anastomoses were made extracorporeally.

Unlike the method of routine resections of abdominal organs, an important feature of the autoflaps' forming is the requirement of adequate perfusion of transplanted tissue flap, which has an artery and a vein within its vascular pedicle, while preserving blood circulation and function of the donor organs.

Postoperative care of patients with transplanted visceral flaps was similar to cases with casual abdominal surgery.

Case report (Fig. 3):

Patient X., 29 years old, case record number OA-3600 with diagnosis: stage II (T2NOM0) tongue cancer, condition after combined treatment in 2004. Histology study \#918/04 showed invasive highly

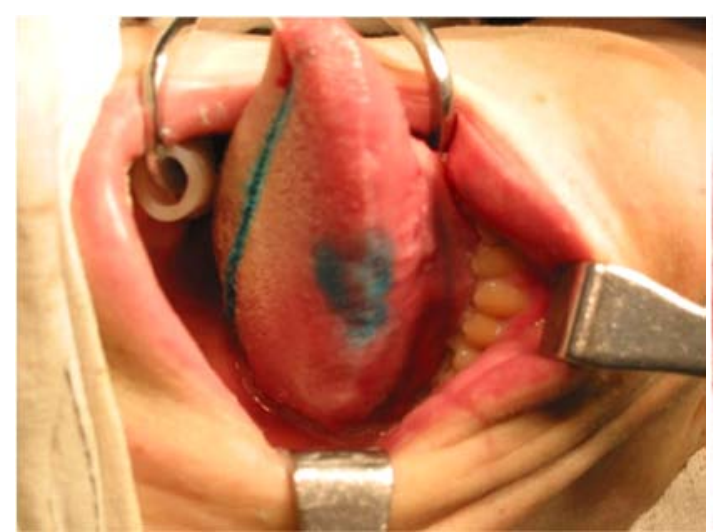

(a)

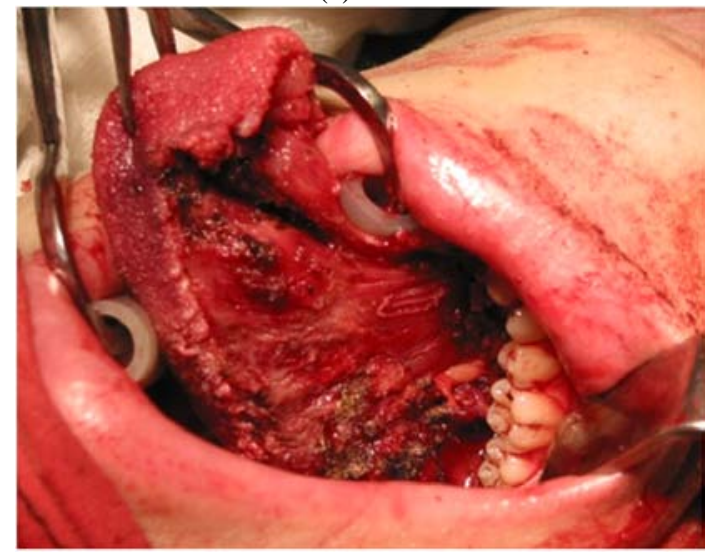

(c) differentiated squamous cell cancer.

Anamnesis: The disease started in Feb. 2004, when the patient first started to experience pain and ulceration in the right part of the tongue. The patient attended the P.A. Hertzen Moscow Cancer Research Institute. Ulcerated tumor of $2 \mathrm{~cm}$ in diameter in the middle third of the lateral part of the tongue was revealed on the examination. Histology study \#918/04 showed invasive highly differentiated squamous cell cancer. Regional lymphatic nodes were not involved. No data confirming regional or distant metastases were found during further complex examination. This case was discussed at the consultation with surgeons, radiologists and chemotherapists. Based on localization, histological structure and extent of tumor, the combined treatment plan was approved, with chemo radiotherapy to be applied during the 1st stage of the treatment. From Aug. 17, 2004 to Sep. 28, 2004 the patient underwent hospital treatment in the Radiation

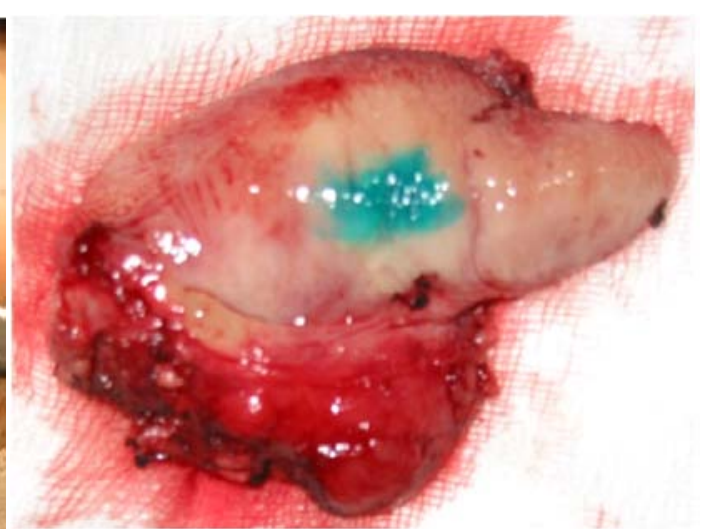

(b)

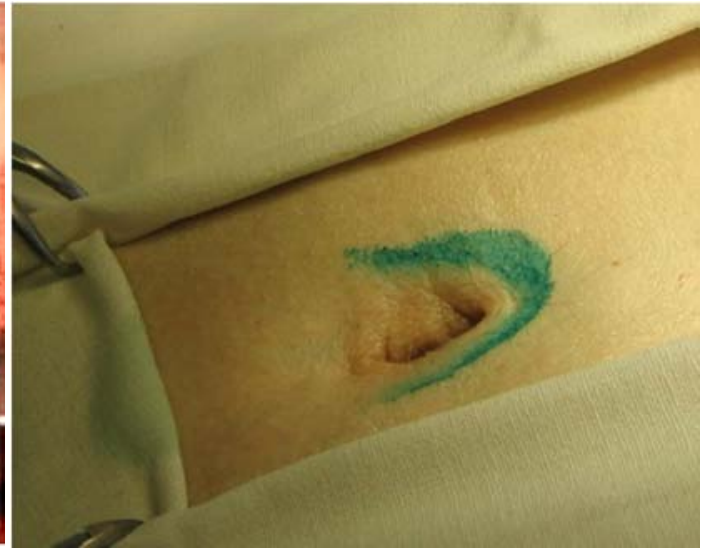

(d) 


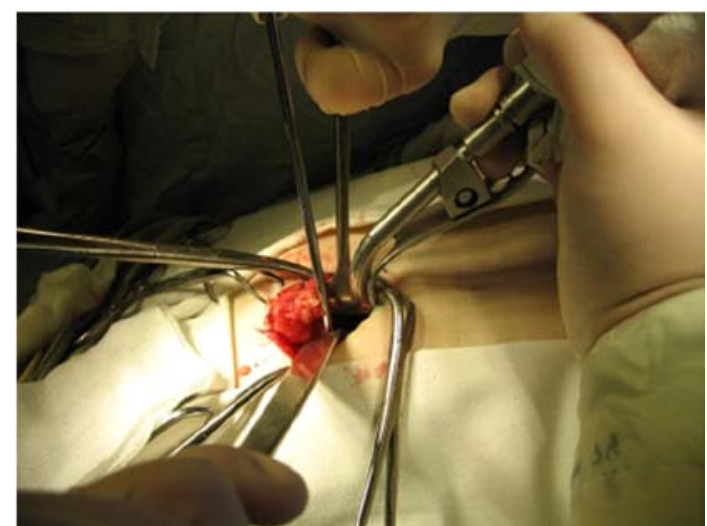

(e)

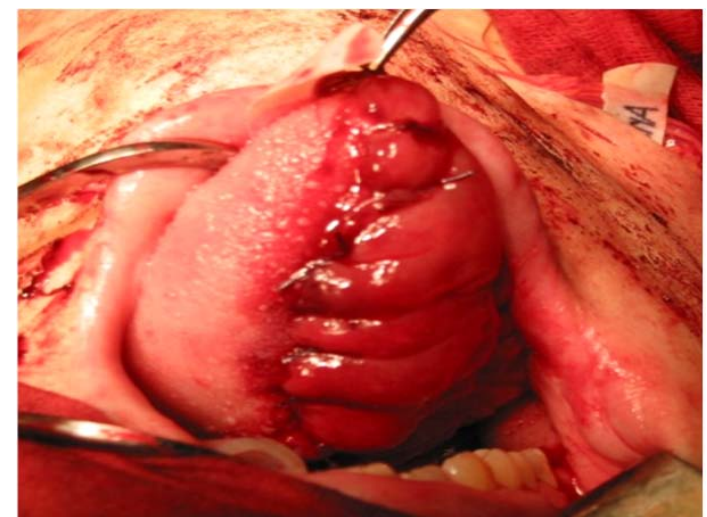

(g)

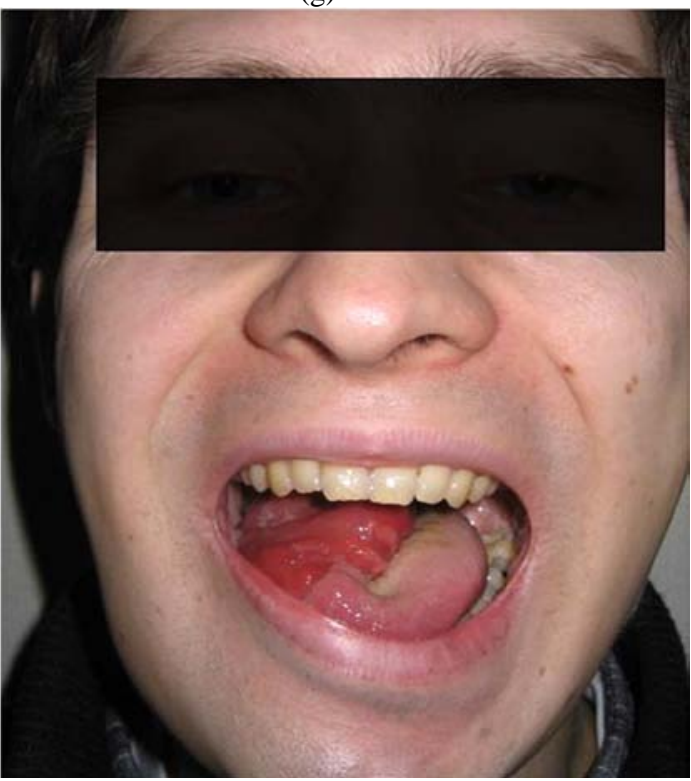

(i)

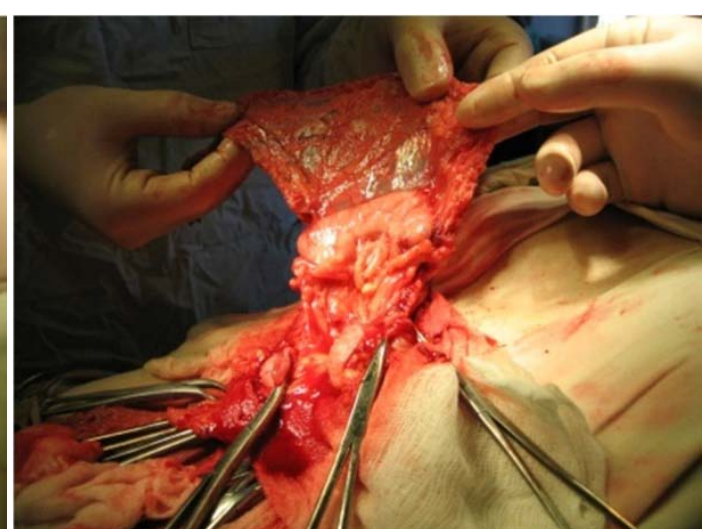

(f)

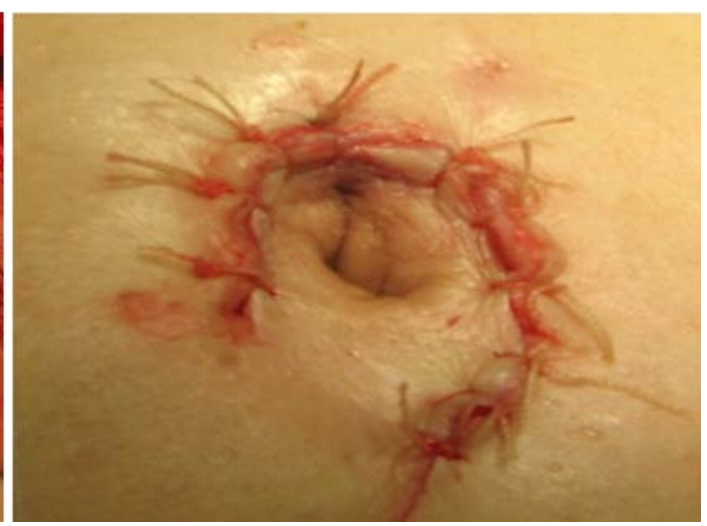

(h)

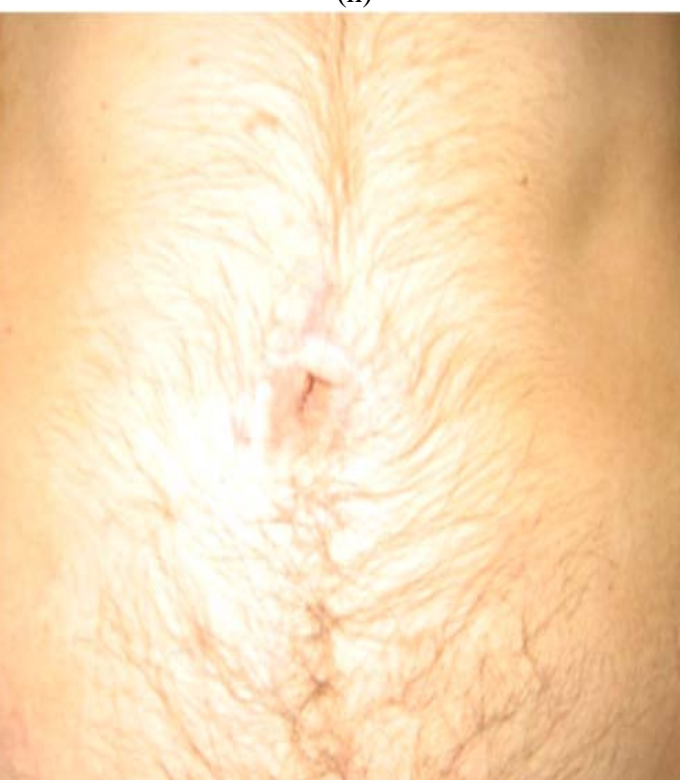

(j)

Fig. 3 Clinical example of microsurgical tongue plastic with colonic-omental flap, performed through a minilaparatomic approach. (a) Tongue resection borders; (b) Removed specimen; (c) Tongue after resection; (d) Marking of the periomphalic incision; (e) Revision of the abdominal cavity through a mini-laparatomic approach; (f) Extracorporeal forming of colonic-omental flap; (g) Colonic part of the flap is fixed in a position of resected right half of the tongue; (h) Final view of a minlaparatomic wound; (i) View of colonic mucosa in the oral cavity 6 months after surgery; (j) Scar on the anterior abdominal wall 6 months after surgery. 
therapy department, where he received with 5-FU and platinum, with the total boost dose of $22 \mathrm{~Gy}$ (finished on Sep. 3, 2004). Treatment was terminated at this dose because of severe radiation reactions. After reactions healing, at the 2nd stage of the combined treatment, a right hemiglossectomy with submandibular lymph node dissection on the right, microsurgical reconstruction using colono-omentalis free flap and tracheostomy were performed on Sep. 29, 2004.

Lower tracheostomy was performed on the IV semiring level under local anesthesia. The Portex No. 8 tracheostomy tube was inserted. Inhalation anesthesia with artificial lung ventilation was started. Through a transoral approach, resection of the right half of the tongue and mucosa of the floor of the mouth was performed using electrosurgical equipment. Skin incision was performed in right submandibular area. Submandibular salivary gland with surrounding fat tissue was mobilized and removed. Right facial artery and vein were mobilized and prepared for further anastomosis. After that, a periomphalic incision was made. Minilaparotomy was performed under video-assisted control by means of endoscopic equipment. Examination of abdominal cavity revealed no pathologic changes. The transverse colon and greater omentum were exteriorized though the minilaparotomy opening on the anterior abdominal wall. Visceral peritoneum was dissected over the site of middle colonic vessels. Vascular pedicle of the flap, containing middle colonic arteria and vein, was mobilized. Vascular clip was placed on the pedicle. After making sure that the collateral circulation in the transverse colon is adequate, the clip was removed. Considering the location of the ramus of collaterals going to the greater omentum from colonic vessels, $10 \mathrm{~cm}$ part of the transverse colon was cut using automatic suturing device, additionally a middle part of the greater omentum was included in the flap. After cutting the supplying vessels of the flap, the flap was placed on the facial defect. The vascular pedicle and the omental part of the flap were introduced through the transmuscular tunnel in the floor of mouth to submandibular area, where recipient vessels are located. The flap was revascularized by creating the end-to-end type anastomosis between the middle colic artery and vein and the right facial artery and vein, under optical magnification. After restarting blood circulation, the viability of the flap was restored. The colonic part of the flap was dissected along the antimesenteric margin and fixed around the borders of the tongue defect and floor of the oral cavity on the right. A vascular anastomosis zone in submandibular area was covered with the omental part of the flap.

The end-to-end type anastomosis with two rows of sutures between parts of the transversal colon was created simultaneously. The abdominal cavity is drained and sutured tightly.

No complications were observed during the postoperative period. Wounds healed by primary intention. The flap is alive, adapted, without any signs of circulation disturbances. In the early postoperative period, patient was fed via naso-gastral feeding tube. Per oral feeding was started on day 14. Tracheal cannula was removed on day 16 . The patient was discharged from the hospital in a satisfactory condition. No signs of recurrence were found during control examination after 6 years. The patient is eating by mouth to the full extent, has no dyspeptic syndrome and gained body weight. Currently, the patient is under dynamic monitoring.

\section{Results}

In 50 cases, the minilaparotomy was successful (3 of these patients had a history of abdominal surgery). Twenty six omental flaps, seven gastro-omental flaps, fifteen colonic-omental flaps and five small intestinal flaps were formed and prepared for transplantation.

In three patients, standard upper-medial laparatomy was performed after revision of abdominal cavity organs. The reason for an extension of the surgical approach was the presence of severe peritoneal adhesions revealed during an attempt to form 1 gastro- 
Table 2 Type of the transplant and different complications.

\begin{tabular}{llll}
\hline Autotransplant & Total & Donor wound & Recipient wound \\
\hline Omental & 26 & - & Flap necrosis-1 $(0.2 \%)$ \\
Gasto-omental & 7 & - & Flap margin necrosis-1 $(0.2 \%)$ \\
Colono-omental & 15 & - & - \\
Small intestinal & 5 & - & Marginal necrosis of the omental part of the flap-1 (0.2\%) \\
Total & 53 & 0 & - \\
\hline
\end{tabular}

omental flap, 1 omental flap and 1 colono-omental flap. The surgery was also successful in these cases, as the flaps were formed and transplanted on recipient wounds.

No intra- or postoperative complications associated with abdominal organs were observed. Three patients in the postoperative period were observed in the transplanted ischemic flap. One patient developed complete necrosis of the flap, two segmental necrosis (Table 2).

\section{Discussion}

Our own experience in creating visceral autotransplants using video-assisted equipment for their subsequent microsurgical revascularization with the purpose of reconstruction of organs and tissues, shows that the method can be successfully used in clinical oncology. According to the case analysis (Table 1), the minimal invasive approach was mostly indicated in young patients willing to avoid an additional scar.

The same opinion we met in other publications about privileges of miniinvasive access for removal of inflamed organs of abdominal cavity as gall bladder, appendicitis etc. [5, 6].

The discussion about readaptation of microsurgically transferred tissues at recipient site is still continuing [7]. Clinical and morphological comparisons did not demonstrate any reliable signs of morphological and functional changes in the mucosa of gastric and colonic fragments after their one-year persistence in the oral cavity or pharynx.

\section{Conclusions}

The use of minimally invasive techniques to form visceral autotransplants for reconstruction of head and neck organs, provides significant reduction of surgical trauma and shortening of treatment duration. This method should be indicated in young patients' willing to avoid an additional scar in the donor area.

\section{References}

[1] V.N. Vavilov, N.V. Kalakutsky, V.S. Ushakov, The immediate results of replacement of extensive head and neck defects using grafts with axial blood supply, in Problems of Microsurgery, in: Abstracts V Symposium of Plastic and Reconstructive Surgery, Moscow, Russia, Oct. 15-16, 1994.

[2] N.D. Stafford, S. Stewart, N. Waterbouse, Cancer of the head and neck, Surgery 18 (1992) 187-192.

[3] V.I. Chissov, Principled Approach to Functional-Sparing Treatment of Patients with Malignant Tumors, P.A. Hertzen MCRI, Moscow, 1991, pp. 5-9.

[4] I.V. Reshetov, V.I. Chissov, Plastic and Reconstructive Microsurgery in Oncology, Goetar-Media, Moscow, 2001, p. 5.

[5] N.J. Switzer, R.S. Gill, S. Karmali, The evolution of the appendectomy: From open to laparoscopic to single incision, Scientifica (Cairo), DOI: 10.6064/2012/895469, Published Online: May 27, 2012, http://dx.doi.org/10.6064/2012/895469 (accessed May 27, 2012).

[6] M. Chalkoo, S. Ahangar, A.M. Durrani, S. Chalkoo, M.J. Shah, M.I. Bashir, Mini-lap cholecystectomy: Modifications and innovations in technique, Int. J. Surg. 8 (2) (2010) 112-117.

[7] C. Wong, F. Wei, Microsurgical free flap in head and neck reconstruction, Head \& Neck 32 (9) (2010) 1236-1245. 